\title{
Does cytomegalovirus load predict the outcome of acute severe ulcerative colitis?
}

\author{
You Sun Kim \\ Department of Internal Medicine, Seoul Paik Hospital, Inje University College of Medicine, Seoul, Korea
}

\section{Article: High mucosal cytomegalovirus DNA helps predict adverse short-term outcome in acute severe ulcerative colitis (Intest Res 2021;19:438-447)}

The treatment of acute severe ulcerative colitis (ASUC) remains a challenge to clinicians, although there have been many developments in therapeutic modalities. ${ }^{1-3}$ The first-line therapy of ASUC is intravenous steroids; however, approximately $15 \%$ to $52 \%$ of ASUC patients require more potent therapy or surgery if there is no response to steroid treatment. ${ }^{4}$ Therefore, predicting the initial responsiveness to steroids is very important for improving the short-term outcome of ASUC patients.

The association between cytomegalovirus (CMV) reactivation and steroid resistance in patients with ulcerative colitis (UC) is well-known and is considered a predictive factor of poor prognosis. Several studies have reported a high prevalence of CMV reactivation in patients with moderate-to-severe UC. In a Korean multicenter prospective study, the prevalence of CMV reactivation was 43\% (31/72) among patients with moderate-to-severe UC and increased to $67 \%(14 / 21)$ in steroid-refractory UC. ${ }^{5}$ Another study also reported that approximately $33.6 \%$ of ASUC patients were diagnosed with CMV reactivation. ${ }^{6}$ Most cases of CMV reactivation occur in patients treated with steroids and/or immunosuppressants, leading to steroid resistance, suggesting that CMV reactivation acts as a true pathogen. On the other hand, CMV reactivation might be

Received August 30, 2021. Accepted September 10, 2021

Correspondence to You Sun Kim, Department of Internal Medicine, Seoul

Paik Hospital, Inje University College of Medicine, 9 Mareunnae-ro, Jung-gu, Seoul 04551, Korea. Tel: +82-2-2270-0012, Fax: +82-2-2270-0257, E-mail:

yousunk69@korea.com regarded as an innocent bystander. Indeed, such reactivation can be identified even in steroid-naïve UC patients, indicating that severe inflammation of the colonic mucosa itself is an important trigger for $\mathrm{CMV}$ reactivation.

In this issue of Intestinal Research, Jain et al. ${ }^{7}$ investigated the role of quantitative CMV polymerase chain reaction (PCR) as a predictor of short-term outcomes in patients with ASUC. Using multivariate analysis, they reported that colonic mucosal CMV DNA load $>2,000$ copies $/ \mathrm{mg}$ (odds ratio, 10.2; 95\% confidence interval $[\mathrm{CI}], 2.6-39.7 ; P<0.01)$ on admission was an independent predictor of steroid failure. Interestingly, positive immunohistochemical (IHC) staining for CMV $(16 \%, P=0.41)$ or CMV PCR positivity $(59 \%, P=0.15)$ were not clinically significant in predicting steroid failure. The authors suggested that, using the receiver operating characteristic curve, the cutoff value of colonic mucosal CMV DNA load $>2,000$ copies/mg was a predictor of steroid failure in ASUC (sensitivity $67 \%$, specificity $70 \%$, area under the curve $74 \%){ }^{7}$ A similar study in a French cohort reported that a CMV DNA load $>250$ copies/ $\mathrm{mg}$ in colonic tissue was a predictor of steroid resistance (likelihood ratio $=4.33$; area under the receiver operating characteristic curve $=0.85){ }^{8}$. They suggested that early antiviral treatment is needed for patients with a high CMV DNA load. Although the cutoff value was different (CMV DNA load $>2,000$ copies/mg vs. CMV DNA load $>250$ copies $/ \mathrm{mg}$ ) between these 2 studies due to different study populations and definition of steroid resistance, the concept is identical in that high CMV 
DNA load is a strong predictor of steroid resistance. ${ }^{7.8}$

The Korean multicenter UC CMV cohort study (median follow-up, $43.35 \pm 20.92$ months) investigated the long-term prognosis of CMV reactivation. The cumulative colectomy (log-rank $P=0.003)$ and disease flare-up rates (log-rank $P=0.048)$ were significantly higher in the CMV-positive group than in the negative group. ${ }^{9}$ Moreover, Lee et al. ${ }^{6}$ reported that ASUC patients with CMV reactivation needed more rescue therapy (2.28-fold higher than CMV negative group). A meta-analysis concluded that inflammatory bowel disease patients with CMV reactivation had a poor prognosis than those without CMV in aspects of steroid failure (relative risk [RR], 1.24; 95\% CI, 1.02-1.49; $P=0.029)$, the colectomy rate (RR, 2.13; 95\% CI, 1.03-4.40; $P=0.042)$ and the incidence of severe inflammatory bowel disease (RR, 1.32; 95\% CI, 1.04-1.67; $P=0.022) .{ }^{10}$ These results definitely suggest that, if ASUC patients do not respond to steroids, they should be evaluated for CMV reactivation.

The gold standard for the diagnosis of CMV reactivation is histopathology with IHC staining (sensitivity 78\%-93\%). Recently, however, quantitative CMV PCR using tissue specimens showed high sensitivity (92.0\%-96.7\%) and specificity (93.0\%98.7\%). ${ }^{11}$ Although, the specificity of IHC staining (98.7\%) is high compared with the reference of colonic CMV PCR, the sensitivity (23.0\%) is relatively low. Recent European Crohn's and Colitis Organisation guideline suggested that the clinical significance of positive results of colonic tissue PCR without IHC staining remains unclear. Nevertheless, to overcome the low sensitivity of IHC staining, colonic CMV PCR test possibly can be considered as a reliable test tool although there is no definite cutoff levels. ${ }^{12}$

The study of Jain et al. ${ }^{7}$ had several limitations; firstly, antiviral treatment was administered in only 7 patients based on IHC staining rather than colonic CMV DNA load. Secondly, the long-term clinical outcomes of patients with colonic CMV DNA load $>2,000$ copies $/ \mathrm{mg}$ were not provided. However, the results of this study were meaningful in terms of providing the cutoff value of quantitative CMV PCR as an independent indicator of steroid responsiveness in ASUC patients. Therefore, when rapid and accurate diagnosis is needed for CMV reactivation in ASUC patients, the use of colonic mucosal quantitative CMV PCR is promising for improving the prognosis of patients. A large prospective study with treatment decisions based on colonic CMV DNA results is needed to clarify whether early antiviral therapy guided by CMV DNA load will alter the clinical outcome.

\section{ADDITIONAL INFORMATION}

\section{Funding Source}

The author received no financial support for the research, authorship, and/or publication of this article.

\section{Conflict of Interest}

Kim YS is an editorial board member of the journal but was not involved in the peer reviewer selection, evaluation, or decision process of this article. No other potential conflicts of interest relevant to this article were reported.

\section{Author Contribution}

Writing and approval of final manuscript: Kim YS.

\section{ORCID}

Kim YS

https://orcid.org/0000-0002-5156-3458

\section{REFERENCES}

1. Fukuda T, Naganuma M, Kanai T. Current new challenges in the management of ulcerative colitis. Intest Res 2019;17:3644.

2. Ooi CJ, Hilmi I, Banerjee R, et al. Best practices on immunomodulators and biologic agents for ulcerative colitis and Crohn's disease in Asia. Intest Res 2019;17:285-310.

3. Yanagi T, Ushijima K, Koga H, et al. Tacrolimus for ulcerative colitis in children: a multicenter survey in Japan. Intest Res 2019;17:476-485.

4. Turner D, Walsh CM, Steinhart AH, Griffiths AM. Response to corticosteroids in severe ulcerative colitis: a systematic review of the literature and a meta-regression. Clin Gastroenterol Hepatol 2007;5:103-110.

5. Kim YS, Kim YH, Kim JS, et al. The prevalence and efficacy of ganciclovir on steroid-refractory ulcerative colitis with cytomegalovirus infection: a prospective multicenter study. J Clin Gastroenterol 2012;46:51-56.

6. Lee HS, Park SH, Kim SH, et al. Risk factors and clinical outcomes associated with cytomegalovirus colitis in patients with acute severe ulcerative colitis. Inflamm Bowel Dis 2016; 22:912-918.

7. Jain S, Namdeo D, Sahu P, et al. High mucosal cytomegalovirus DNA helps predict adverse short-term outcome in acute severe ulcerative colitis. Intest Res 2021;19:438-447.

8. Roblin X, Pillet S, Oussalah A, et al. Cytomegalovirus load in inflamed intestinal tissue is predictive of resistance to immu- 
nosuppressive therapy in ulcerative colitis. Am J Gastroenterol 2011;106:2001-2008.

9. Kim YS, Kim YH, Kim JS, et al. Long-term outcomes of cytomegalovirus reactivation in patients with moderate to severe ulcerative colitis: a multicenter study. Gut Liver 2014;8:643647.

10. Zhang WX, Ma CY, Zhang JG, et al. Effects of cytomegalovirus infection on the prognosis of inflammatory bowel disease pa- tients. Exp Ther Med 2016;12:3287-3293.

11. Inflammatory Bowel Disease Group, Chinese Society of Gastroenterology, Chinese Medical Association. Evidence-based consensus on opportunistic infections in inflammatory bowel disease (republication). Intest Res 2018;16:178-193.

12. Kucharzik T, Ellul P, Greuter T, et al. ECCO guidelines on the prevention, diagnosis, and management of infections in inflammatory bowel disease. J Crohns Colitis 2021;15:879-913. 